\title{
Coagulação endoscópica do plexo coroide no tratamento primário da hidranencefalia e da hidrocefalia extrema
}

\author{
Leandro Custódio do Amaral', Sérgio Lemos², Thales Eduardo', Hugo Abi-Saber \\ Rodrigues Pedrosa ${ }^{3}$, Sandro Pedroso Lemos ${ }^{3}$, Liz Custódio Souza Seabra
}

Fundação Benjamin Guimarães, Hospital da Baleia, Belo Horizonte, MG, Brasil.

\section{RESUMO}

Objetivos: Descrever a técnica de coagulação neuroendoscópica do plexo coroide e divulgá-la como opção de tratamento primário da hidranencefalia e da hidrocefalia extrema. Mostrar a casuística de coagulação endoscópica de plexo coroide do Serviço de Neurocirurgia do Hospital da Baleia em Belo Horizonte, MG, Brasil. Métodos: Imagens tomográficas foram avaliadas por um neurocirurgião, sendo selecionadas crianças portadoras de hidranencefalia e hidrocefalia extrema com cavidade ventricular única. Os pacientes foram acompanhados por até três anos no pós-operatório. Resultados: Foram tratadas seis crianças com hidranencefalia e seis com hidrocefalia extrema. Um paciente faleceu na primeira semana de pós-operatório em função de choque hipovolêmico e hipernatremia graves e outro cursou com aumento progressivo do perímetro cefálico e necessidade de derivação ventriculoperitoneal. Um terceiro paciente apresentou óbito tardio não relacionado à cirurgia endoscópica. Os demais apresentaram evolução adequada no pós-operatório. Conclusão: $A$ coagulação endoscópica do plexo coroide mostrou-se alternativa eficaz no tratamento da hidranencefalia e da hidrocefalia extrema.

\section{PALAVRAS-CHAVE}

Hidranencefalia, hidrocefalia, plexo coroide.

\section{ABSTRACT}

Endoscopic coagulation of the choroid plexus in the primary treatment of extreme hydrocephalus and hydranencephaly

Objectives: Describing the neuroendoscopic technique for coagulation of the choroid plexus and publicize it as an option for the primary treatment of extreme hydrocephalus and hydranencephaly. Display the series of endoscopic coagulation of the choroid plexus of the Neurosurgery Department of the Hospital da Baleia in Belo Horizonte, MG, Brazil. Methods: Tomographic images were used to select children with hydranencephaly and extreme hydrocephalus with single ventricular cavity. Patients were followed for up to three years postoperatively. Results: Six children with hydranencephaly and six with extreme hydrocephalus were treated. One patient died in the first week postoperatively due to hypovolemic shock and severe hypernatremia and another developed progressive increase in head circumference and the need for ventriculoperitoneal shunt. $A$ third patient had late death unrelated to endoscopic surgery. The others had adequate postoperative evolution. Conclusion: The endoscopic coagulation of the choroid plexus is an effective alternative in the treatment of extreme hydrocephalus and hydranencephaly.

\section{KEYWORDS}

Hydranencephaly, hydrocephalus, choroud plexus.

1 Residente de Neurocirurgia da Fundação Benjamin Guimarães, Hospital da Baleia, Belo Horizonte, MG, Brasil.

2 Neurocirurgião, chefe do Serviço de Neurocirurgia da Fundação Benjamin Guimarães, Hospital da Baleia, Belo Horizonte, MG, Brasil.

3 Neurocirurgião e preceptor da residência de Neurocirurgia da Fundação Benjamin Guimarães, Hospital da Baleia, Belo Horizonte, MG, Brasil.

4 Residente de Gastroenterologia Pediátrica do Hospital das Clínicas da Universidade Federal de Minas Gerais (UFMG), Belo Horizonte, MG, Brasil. 


\section{Introdução}

A hidranencefalia e a hidrocefalia extrema são patologias extremamente graves e complexas do ponto de vista médico e socioeconômico. Os pacientes com essas patologias são frequentemente submetidos a derivações ventriculares com inúmeras complicações (fístula liquórica, infecções do sistema nervoso central, disfunções do sistema de derivação e escaras sobre os reservatórios das derivações ventriculoperitoneais). Essas complicações levam à necessidade de múltiplas cirurgias para revisões e/ou trocas dos sistemas de derivações e antibioticoterapias por períodos prolongados com custos bastante elevados para o sistema de saúde. ${ }^{1,2}$ Além disso, a refratariedade a esses tratamentos pode levar ao crescimento exagerado do perímetro cefálico com consequências neurológicas e estéticas desastrosas.

Descrever a técnica de coagulação neuroendoscópica do plexo coroide e divulgá-la como uma opção de tratamento primário da hidranencefalia e da hidrocefalia extrema.

Mostrar a casuística dos 12 casos operados no Serviço de Neurocirurgia do Hospital da Baleia até o momento.

\section{Materiais e métodos}

Foram selecionadas, por meio de imagens tomográficas, crianças portadoras de hidranencefalia ou hidrocefalia extrema com cavidade ventricular única. Os pacientes foram acompanhados no pós-operatório com avaliações clínicas e medidas do perímetro periódicas por período de até três anos (Tabela 1).

\section{Resultados}

Foi realizada a coagulação endoscópica do plexo coroide em seis crianças com hidranencefalia e seis com hidrocefalia extrema. Um paciente faleceu na primeira semana de pós-operatório em função de choque hipovolêmico e hipernatremia graves e refratários às medidas clínicas. Outro paciente cursou com aumento progressivo do perímetro cefálico com necessidade de derivação ventriculoperitoneal dois meses após o procedimento endoscópico para coagulação do plexo coroide. Todos os outros pacientes apresentaram adequada evolução pós-operatória, sem necessidade de qualquer outro tratamento cirúrgico, complicações graves relacionadas ao procedimento ou aumento progressivo do perímetro cefálico (Tabela 2).

\begin{tabular}{|c|c|c|}
\hline \multicolumn{3}{|c|}{$\begin{array}{c}\text { Tabela } 1 \text { - Perfil dos pacientes submetidos } \\
\text { à coagulação de plexo coroide }\end{array}$} \\
\hline Diagnóstico tomográfico & Hidranencefalia & $\begin{array}{c}\text { Hidrocefalia } \\
\text { extrema }\end{array}$ \\
\hline Pacientes & 6 & 6 \\
\hline \multicolumn{3}{|l|}{ Sexo } \\
\hline Masculino & 5 & 5 \\
\hline Feminino & 1 & 1 \\
\hline Idade média na cirurgia (dias) & 97,3 & 89,4 \\
\hline Perímetro cefálico médio $(\mathrm{cm})$ & 43,7 & 50,9 \\
\hline
\end{tabular}

\begin{tabular}{lcc}
\hline \multicolumn{3}{c}{ Tabela 2 - Resultados e complicações da } \\
coagulação de plexo coroide \\
\hline Resultados e complicações & Pacientes & Porcentagem \\
\hline Controle satisfatório do perímetro cefálico & $9 / 10$ & $90,0 \%$ \\
Distúrbios hidroeletrolíticos & $2 / 12$ & $16 \%$ \\
Infecção do sistema nervoso central & $1 / 12$ & $8,3 \%$ \\
Crises convulsivas pós-operatórias & $5 / 12$ & $41,6 \%$ \\
Óbito relacionado ao procedimento & $1 / 12$ & $8,3 \%$ \\
Óbito tardio e não relacionado & $1 / 12$ & $8,3 \%$ \\
ao procedimento & & \\
\hline
\end{tabular}

\section{Discussão}

A diferenciação de hidranencefalia e hidrocefalia extrema nem sempre é fácil e é objeto de discussão. A hidranencefalia é uma rara e grave malformação encefálica que ocorre na fase pós-neurulação. Pode ter causa vascular, devido a infartos bilaterais nos territórios das artérias carótidas internas, com consequente ausência de tecido cerebral nos territórios das artérias cerebrais anteriores e médias e preservação nos territórios das artérias cerebrais posteriores. Além disso, a hidranencefalia pode estar associada a infecções como herpes congênito ou neonatal e toxoplasmose, fatores genéticos e/ou exposição às substâncias tóxicas. ${ }^{2} \mathrm{~A}$ ausência das estruturas supratentoriais perfundidas pelas artérias carótidas permite aumento da porção da cavidade craniana preenchida por liquor. Apresenta-se por déficit cognitivo grave, tetraparesia espástica e macrocrania progressiva com sinais de hipertensão intracraniana. $\mathrm{O}$ prognóstico é pobre e a maioria dessas crianças falece na primeira infância (Figura 1).

Hidrocefalia extrema é definida como o aumento muito acentuado de todo o sistema ventricular e assim com a hidranencefalia apresenta cavidade ventricular única supratentorial com ausência de septo pelúcido, mas com um fino manto cortical (Figura 2). 


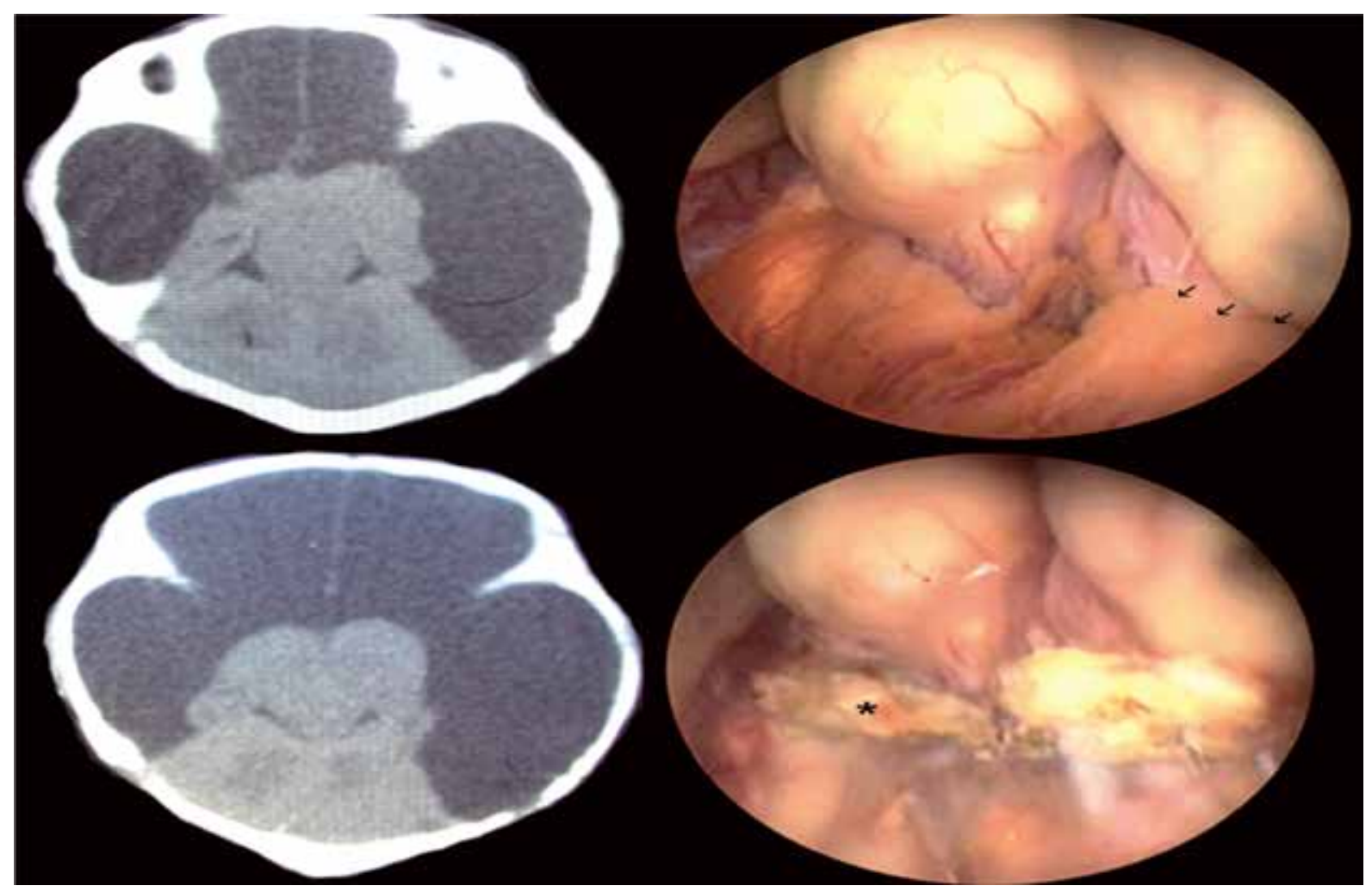

Figura 1 - Tomografia evidenciando a ausência das estruturas supratentoriais em lactente com hidranencefalia com manutenção apenas do diencéfalo e dos núcleos da base. Visão endoscópica intraoperatória dos plexos coroides antes e após a coagulação, apontados respectivamente pelas setas e pelo asterisco.

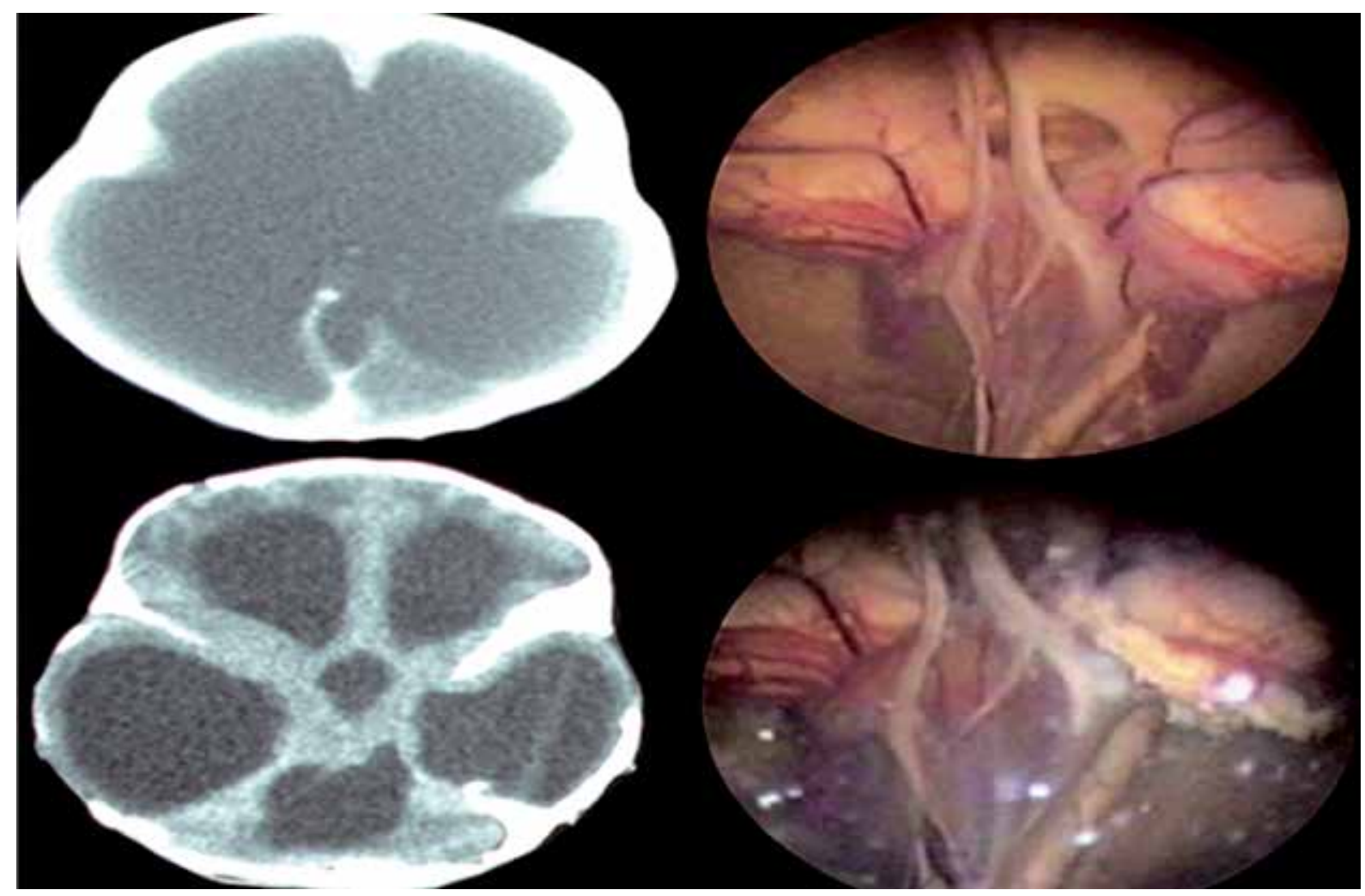

Figura 2 - À esquerda, tomografias de lactente com hidrocefalia extrema mostrando córtex frontal e terceiro ventrículo. À direita, visão endoscópica intraoperatória antes e após a coagulação do plexo coroide. 
A manutenção apenas de pequena quantidade de parênquima cerebral occipital associada à ausência de atividade eletroencefalográfica fala a favor de hidranencefalia. Já a presença de fino manto cortical frontal associado a um traço eletroencefalográfico de base, mesmo que anormal, sugere hidrocefalia extrema. ${ }^{3}$

Ambas as condições necessitam de tratamento em decorrência de aumento progressivo e acentuado do perímetro cefálico e sinais de hipertensão intracraniana. Classicamente, pacientes com essas patologias têm sido submetidos a implantes de sistemas de derivações ventriculoperitoneais visando à expansão do manto cortical nos pacientes com hidrocefalia extrema e ao controle do perímetro cefálico em ambos os casos. Contudo, a coagulação endoscópica dos plexos coroides tem sido cada vez mais utilizada na tentativa de atingir esses objetivos com índices de morbimortalidade mais reduzidos. ${ }^{1,4,5}$

A técnica para realização da coagulação de plexo coroide consiste em, com paciente sob anestesia geral e em posição supina neutra, realizar incisão semicircular na borda posterior da fontanela anterior ao nível da linha pupilar média e abrir pequena janela óssea seguida pela abertura longitudinal da dura-máter e introdução do neuroendoscópio rígido de zero grau. Posteriormente, são realizados o reconhecimento das estruturas cerebrais e a identificação do plexo coroide bilateralmente, utilizando como solução de irrigação o ringer simples. Em uso do cateter bipolar, os plexos coroides são amplamente coagu- lados no sentido do forame de Monro até a face inferior do tálamo (de proximal para distal), sendo iniciada a coagulação pelo lado direito. Nos casos em que é possível penetrar no terceiro ventrículo e identificar as estruturas do assoalho deste, é realizada a terceiroventriculocisternostomia (cinco casos de hidrocefalia extrema) e, ao final do procedimento, a cavidade ventricular é preenchida com solução de ringer a fim de evitar pneumoencéfalo excessivo e o "desabamento" do crânio no pós-operatório imediato. Por fim, a dura-máter é suturada hermeticamente com enxerto de pericrânio, a janela óssea fixada no periósteo e a pele fechada em dois planos. O tempo médio de cirurgia é de aproximadamente 60 minutos.

Os altos índices de controle satisfatório do perímetro cefálico, perfazendo $90 \%$ dos casos, associados à relativa baixa mortalidade $(16,6 \%)$, são fatores que falam a favor do uso da coagulação endoscópica do plexo coroide como tratamento da hidranencefalia e da hidrocefalia extrema. Vale descartar que um desses óbitos ocorreu em paciente grave que foi submetido ao procedimento endoscópico após os seis meses de vida. Além disso, grande parte das complicações relacionadas à coagulação endoscópica do plexo coroide, como crises convulsivas pós-operatórias $(41,6 \%)$, infecções do sistema nervoso central $(8,3 \%)$ e parte dos distúrbios hidroeletrolíticos $(16,6 \%)$, pôde ser completamente revertida com tratamento clínico. Um paciente faleceu em decorrência de distúrbio hidroeletrolítico grave (Figura 3).

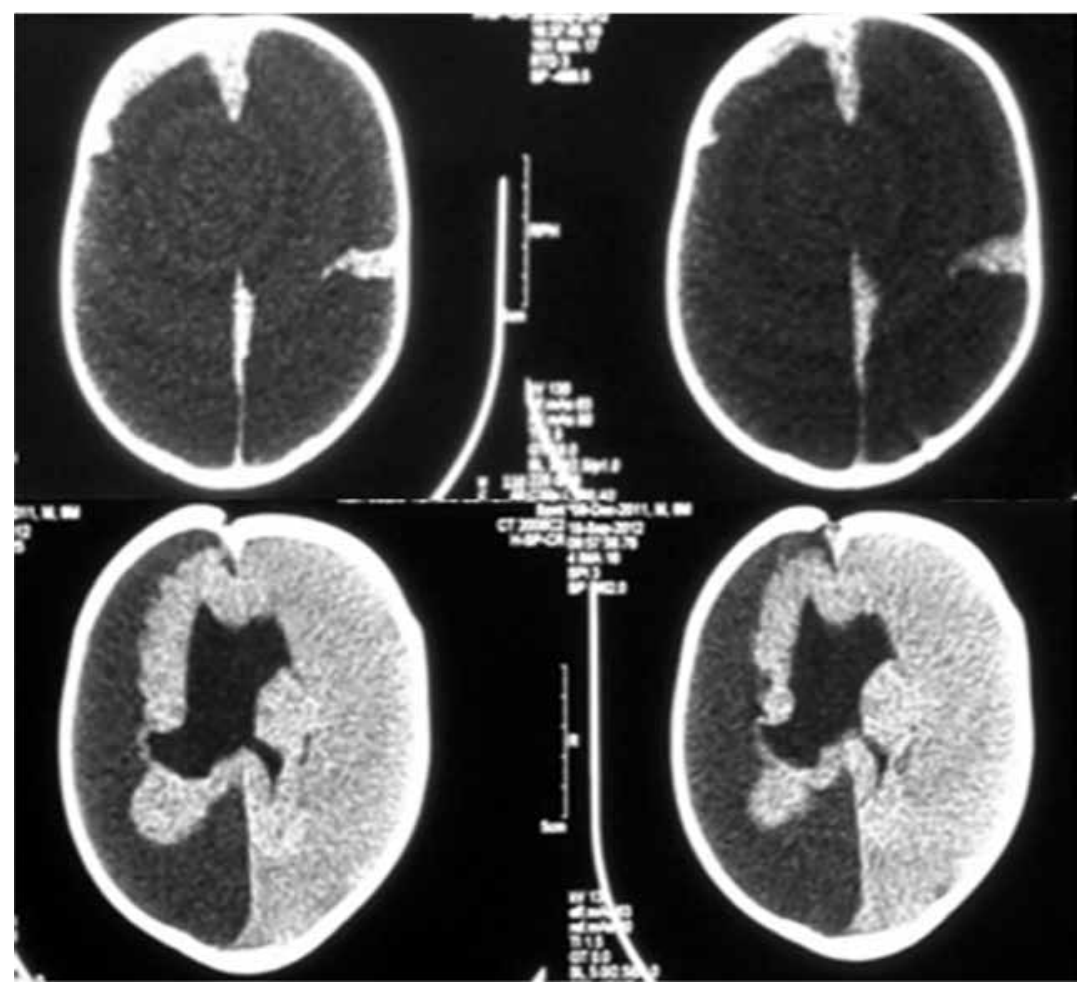

Figura 3 - Tomografia pré- e pós-operatória tardia (sete meses) de criança com hidrocefalia extrema submetida à coagulação dos plexos coroides è terceiroventriculostomia que evidencia melhora acentuada da hidrocefalia com expansão do manto cerebral. 


\section{Conclusão}

A coagulação endoscópica do plexo coroide, apesar de passível de complicações, é uma alternativa viável e eficaz no controle do crescimento do perímetro cefálico e evita várias complicações associadas aos sistemas de derivações ventriculares, reduzindo consideravelmente o número de internações e cirurgias para troca de próteses e, por consequência, os custos do sistema de saúde. Porém, para atingir resultados satisfatórios, é essencial que haja treinamento especializado, além de uma seleção criteriosa dos pacientes e bom suporte clínico pós-operatório.

Ainda são necessários novos estudos com casuísticas e períodos de acompanhamento dos pacientes maiores.

\section{Conflitos de interesse}

Os autores declaram não haver conflitos de interesse.

\section{Referências}

1. Malheiros JA, Trivelato FP, Oliveira MM, Gusmão S, Cochrane DD, Steinbok P. Endoscopic choroid plexus cauterization versus ventriculoperitoneal shunt for hydranencephaly and near hydranencephaly: a prospective study. Neurosurgery. 2010;66(3):459-64.

2. Albright L. Percutaneous choroid plexus coagulation in hydranencephaly. Childs Brain. 1981;8(2):134-7.

3. linuma K, Handa I, Kojima A, Hayamizu S, Karahashi M. Hydranencephaly and maximal hydrocephalus: usefulness of electrophysiological studies for their differentiation. $J$ Child Neurol. 1989;4(2):114-7.

4. Shitsama S, Wittayanakorn N, Okechi H, Albright AL. Choroid plexus coagulation in infants with estreme hydrocephalus and hydranenecephaly. J Neurosurg Pediatr. 2014;14(1):55-7.

5. Sandberg DI, Chamiraju P, Zoeller G, Bhatia S, Ragheb $J$. Endoscopic choroid plexus coagulation in infants with hydranencephaly or hydrocephalus with a minimal cortical mantle. Pediatr Neurosurg. 2012;48 (1):6-12.

Endereço para correspondência

Leandro Custódio do Amaral

Rua Grão Pará, 638, ap. 600

Bairro Santa Efigênia

30150-341 - Belo Horizonte, MG, Brasil

E-mail: leandroamaral_ns@hotmail.com 